\title{
X-ray Absorption Intensity at High-energy Region
}

\author{
Takashi Fujikawa $^{\text {a }}$, Katsumi Kaneko ${ }^{\text {b }}$ \\ ${ }^{a}$ Graduate School of Advanced Integration Science, Chiba University, 1-33 \\ Yayoi-cho, Inage-ku, Chiba 263-8522, Japan \\ ${ }^{\mathrm{b}}$ Exotic Nanocarbpon Research Center, Shinsyu University, Wakasato \\ 4-17-1, Nagano 380-8553, Japan
}

\begin{abstract}
We theoretically discuss X-ray absorption intensity in high-energy region far from the deepest core threshold to explain the morphology-dependent mass attenuation coefficient of some carbon systems, carbon nanotubes (CNTs), highly oriented pyrolytic graphite (HOPG) and fullerenes $\left(\mathrm{C}_{60}\right)$. The present theoretical approach is based on the many-body X-ray absorption theory including the intrinsic losses ( shake-up losses ). In the high-energy region the absorption coefficient has correction term dependent on the solid state effects given in terms of the polarization part of the screened Coulomb interaction $W_{p}$. We also discuss the tail of the valence band X-ray absorption intensity. In the carbon systems C 2s contribution has some influence on the attenuation coefficient even in the high energy region at $20 \mathrm{keV}$.
\end{abstract}

Key words: High-energy X-ray absorption, Thomas-Reiche-Kuhn sum rule, intrinsic loss, core-hole screening,

\section{Introduction}

X-ray absorption intensity in high-energy region has extensively been studied for atoms and molecules $[1,2]$. The dependence on the photon energy $\omega$ is on average between $\omega^{-7 / 2}$ and $\omega^{-8 / 3}$ in accord with empirical data on X-ray absorption. Berkowitz has interests in confirming the Thomas-Reiche-Kuhn sum rule [1], so that they try to measure the X-ray absorption intensity even in the high energy region. In his book, for example, Berkowitz obtained the absolute photoabsorption spectrum of $\mathrm{C}_{60}$ from the data by Itchkawitz et al.

Email address: tfujikawa@faculty.chiba-u.jp (Takashi Fujikawa). 
[3] normalized to the summed atomic cross sections, where he assume that the photoabsorption cross section is simply given by the sum of atomic cross sections without solid state effects. In the approximation we assume that the electric dipole transition still works even in the high-energy region. The sum rule says that the integrated oscillator strength is just as same as the number of all the composite electrons [1]. In the visible and ultraviolet region, diamond and graphite show quite different photoabsorption spectra, and they also show quite different X-ray absorption near edge structures (XANES). So that the integrated intensity from 0 to some high energy photon $\omega$ should be different for these systems. Above that energy, the X-ray absorption cross section shows smooth decay. To satisfy the sum rule, those smoothly decaying function should be dependent on the systems. This consideration helps us to understand the solid state effects on the high-energy X-ray absorption cross section dependent on their morphology. We however have no idea about how large they are.

For condensed systems such systematic studies have not been undertaken except near edge regions, where X-ray absorption fine structures are now most powerful techniques to study geometric, electronic and also magnetic structures of ordered and even disordered systems. In the energy region far from the edge the X-ray absorption spectra can have some useful information. Kaneko and his coworkers have observed that the mass attenuation coefficient of carbon nanotubes (CNTs) is significantly larger than that observed for highly oriented pyrolytic graphite $(\mathrm{HOPG})$ and fullerenes $\left(\mathrm{C}_{60}\right)$ by 20$50 \%$ for monochromatic Mo $\mathrm{K}_{\alpha}$ line $(\omega-20 \mathrm{keV})$ [4]. The attenuation coefficient of CNTs increases by reducing the sample thickness. These observations could not be explained in the framework of the conventional X-ray absorption theory: In the high- energy region, the X-ray absorption intensity should be independent of their morphology.

In the present work we discuss the X-ray absorption intensity far from the edges where elastic scatterings of photoelectrons plays a very minor role. Our theoretical approach is based on the many-body X-ray absorption theory including the intrinsic losses ( shake-up losses ). For solids composed of only light elements such as $\mathrm{C}, \mathrm{N}, \mathrm{O}, .$. , the tail of the valence level photoabsorption intensity can contribute to the X-ray absorption intensity in high energy region.

\section{Basic Theory}

The X-ray absorption intensity $I(\omega)$ for a photon of angular frequency $\omega$ is represented in terms of the initial and final many-body states $\mid 0>$ and $\mid f>$ 


$$
I(\omega)=2 \pi \sum_{f}|<f| H_{e p}|0>|^{2} \delta\left(E_{0}+\omega-E_{f}\right)
$$

where $H_{e p}$ is the electron-photon interaction Hamiltonian

$$
H_{e p}=\int \psi^{\dagger}(x) \Delta(x) \psi(x) d x, x=(\mathbf{r}, \sigma) .
$$

Both many-body scattering theory [5,6] and Keldysh Green's function approaches $[7,8]$ provides us with a useful formula to describe XAFS spectra

$$
\begin{aligned}
I(\omega) & =-2 \operatorname{Im}<c\left|\Delta^{*} g(\varepsilon) \Delta\right| c>\left|S_{0}\right|^{2} \\
& -2 \sum_{n \neq 0} \operatorname{Im}\left[<c\left|\Delta^{*} g\left(\varepsilon-\omega_{n}\right) V_{n 0} g(\varepsilon) \Delta\right| c>S_{n}^{*} S_{0}\right. \\
& \left.+<c\left|\Delta^{*} g(\varepsilon) V_{0 n} g\left(\varepsilon-\omega_{n}\right) \Delta\right| c>S_{n} S_{0}^{*}\right] \\
& -2 \sum_{n \neq 0} \operatorname{Im}<c\left|\Delta^{*} g\left(\varepsilon-\omega_{n}\right) \Delta\right| c>\left|S_{n}\right|^{2}-\ldots
\end{aligned}
$$

where $V_{n m}$ is the extrinsic loss amplitude for the transition $m^{*} \rightarrow n^{*}$, and $\varepsilon=\omega+E_{0}-E_{0}^{*}$ is the kinetic energy of photoelectrons, $\omega_{n}=E_{n}^{*}-E_{0}^{*}$ is the excitation energy of the target with core hole. The first term describes the the main absorption band without losses, the second describes the interference between intrinsic and extrinsic losses, the third the intrinsic losses, and so on. As well known in XAFS spectra large-angle scatterings of photoelectrons are unimportant: Far from the edge in the high-energy region, the extrinsic losses and elastic scatterings are not so important [6]. We thus expect that the X-ray absorption intensity $-2 \operatorname{Im}<c\left|\Delta^{*} g(\varepsilon) \Delta\right| c>$ shows slow change with photon energy $\omega$. The complicated absorption intensity (3) is simplified in these high-energy region

$$
I(\omega)=-2 \operatorname{Im}<c\left|\Delta^{*} g(\varepsilon) \Delta\right| c>\left[\left|S_{0}\right|^{2}+\sum_{n \neq 0}\left|S_{n}\right|^{2}\right]
$$

where $S_{n}$ describes the intrinsic loss amplitude in the process $0^{*} \rightarrow n^{*}$.

In this approximation the X-ray absorption intensity is simply given by

$$
\begin{aligned}
& I(\omega)=\mu_{c}(\varepsilon), \quad \varepsilon=\omega+E_{0}-E_{0}^{*}, \\
& \mu_{c}(\varepsilon)=-2 \operatorname{Im}<c\left|\Delta^{*} g(\varepsilon) \Delta\right| c>.
\end{aligned}
$$

This is atomic absorption intensity which is not sensitive to solid state effects in the high-energy region, whereas it can basically include solid state and many-body effects through the atomic potential in solids [9]. In the next step we include the effects of the energy difference in eq. (3), 


$$
I(\omega)=\mu_{c}(\omega)-\mu_{c}^{\prime}(\omega) \sum_{n} \omega_{n}\left|S_{n}\right|^{2}-\ldots
$$

In the second term $\mu_{c}^{\prime}(\omega)$ should be negative because $\mu_{c}(\omega)$ is a decreasing function of $\omega$.

\section{Relaxation Energy}

The sum $\sum_{n} \omega_{n}\left|S_{n}\right|^{2}$ is interpreted as the relaxation energy $\Delta E_{c}$ as pointed out by Manne and Åberg [10]. They used $\Delta$ SCF approximation to derive the relation. Here we derive the relation based on more general situation because the $\Delta$ SCF approximation is not easy to describe the collective excitation losses like plasmon losses. To specify the problem, we introduce the Hamiltonian widely used to study deep core processes [15]

$$
H=H_{v}+V_{c} b b^{\dagger}+\varepsilon_{c} b^{\dagger} b .
$$

Here $H_{v}$ is the full many-electron Hamiltonian for valence electrons, $V_{c}$ is the interaction between the core-hole and valence electrons, and $\varepsilon_{c}$ is the core electron energy whose annihilation (creation) operator is $b\left(b^{\dagger}\right)$. The hole state $\mid n_{v}^{*}>$ satisfies the equation

$$
H_{v}^{*}\left|n_{v}^{*}>=E_{n}^{v *}\right| n_{v}^{*}>,\left(H_{v}^{*}=H_{v}+V_{c}\right)
$$

whereas no-hole state $\mid m_{v}>$ satisfies

$$
H_{v}\left|m_{v}>=E_{m}^{v}\right| m_{v}>
$$

In terms of these states and energies, we have

$$
\begin{aligned}
& \sum_{n} \omega_{n}\left|S_{n}\right|^{2}=\sum_{n}\left(E_{n}^{v *}-E_{0}^{v *}\right)\left|<n_{v}^{*}\right| 0_{v}>\left.\right|^{2} \\
& =<0_{v}\left|H_{v}^{*}\right| 0_{v}>-<0_{v}^{*}\left|H_{v}^{*}\right| 0_{v}^{*}>.
\end{aligned}
$$

From the variational principle, the right hand side should be positive. The ionization energy associated with the core ionization is thus given without the relaxation effects

$$
I_{c}=<0_{v}\left|H_{v}^{*}\right| 0_{v}>-<c, 0_{v}|H| c, 0_{v}>
$$


where $\left|c, 0_{v}>=\right| c>\mid 0_{v}>$ is the ground state of the target. On the other hand , the ionization energy $I_{c}^{*}$ taking the relaxation into account is thus given by

$$
I_{c}^{*}=<0_{v}^{*}\left|H_{v}^{*}\right| 0_{v}^{*}>-<c, 0_{v}|H| c, 0_{v}>
$$

The relaxation energy is thus given by

$$
\begin{aligned}
\Delta E_{c} & =I_{c}-I_{c}^{*}=<0_{v}\left|H_{v}^{*}\right| 0_{v}>-<0_{v}^{*}\left|H_{v}^{*}\right| 0_{v}^{*}> \\
& =\sum_{n}\left|S_{n}\right|^{2} \omega_{n} .
\end{aligned}
$$

We furthermore use a simple formula for $\mu_{c}(\omega)$ in the high energy region as given for atoms and molecules $[1,2]$

$$
\mu_{c}(\omega) \propto \omega^{\alpha} \quad(-8 / 3<\alpha<-7 / 2) .
$$

In this case we have a very simple formula to describe the X-ray absorption intensity

$$
\begin{aligned}
I(\omega) & =\mu_{c}(\omega)-\mu_{c}^{\prime}(\omega) \Delta E_{c} \\
& =\mu_{c}(\omega)\left(1+\frac{\alpha \Delta E_{c}}{\omega}\right)
\end{aligned}
$$

Our task is thus to calculate the relaxation energy. In carbon systems, $\Delta E_{c}$ is in the order of $15 \mathrm{eV}$. When the photon energy $\omega$ is $4500 \mathrm{eV}$, the correction term to $\mu_{c}(\omega)$ is in the order of $1 \%$.

We can expect that the relaxation energy $\Delta E_{c}$ should be influenced by solid state effects. In the $G W$ approximation, the relaxation energy $\Delta E_{c}$ associated with the core excitation from the core level $c$ is given by [11]

$$
\begin{aligned}
& \Delta E_{c}=\frac{1}{2} W_{p o l}(\mathbf{0}, \mathbf{0} ; 0) \\
& =\frac{1}{2} \int d \mathbf{r} d \mathbf{r}^{\prime} v(\mathbf{r}) \pi\left(\mathbf{r}, \mathbf{r}^{\prime} ; 0\right) v\left(\mathbf{r}^{\prime}\right)
\end{aligned}
$$

where $W_{p o l}$ is the polarization part of the screened Coulomb propagator, $W_{p o l}=W-v$. The reducible polarization propagator $\pi$ which depends on solid state effects. 


\section{Valence Excitation}

In addition to the X-ray absorption from deep cores, those from extended shallow levels have to some extent finite contribution. Of course complicated multiple scatterings from surrounding atoms are crucial even in the intermediate energy region $(<500 \mathrm{eV})[12,13]$. In high-energy region $(>1000 \mathrm{eV}$ ), the scatterings from surrounding atoms play only minor roles. The interference terms of photoelectron waves emanating from different sites should be suppressed because of the Debye-Waller factors particularly in the highenegy region, thus we obtain Gelius formula for the photoemission and X-ray absorption intensity [14]. The X-ray absorption intensity $I_{e x}$ from extended levels is thus given by the weighted sum of subshell cross section $\mu_{\alpha}$ of $\alpha$ th atomic orbital $(\mathrm{AO})$

$$
I_{e x}(\omega)=\sum_{\alpha} P_{\alpha} \mu_{\alpha}(\omega)
$$

where $P_{\alpha}$ is the population of the $\alpha$ th AO. For example, in diamond $\mathrm{C}$ $2 s$ and $2 p$ X-ray absorption intensity $\mu_{2 s}(\omega)$ and $\mu_{2 p}(\omega)$ are different. The population $P_{2 s}$ and $P_{2 p}$ depend on the systems under consideration. In diamond $P_{2 p} \approx 3$ and $P_{2 s} \approx 1$. At $\omega=5 \mathrm{keV}$, the ratio $\mu_{2 s}(\omega) / \mu_{1 s}(\omega)=0.062$, $\mu_{2 p}(\omega) / \mu_{1 s}(\omega)=0.61 \times 10^{-3}$ [16]: The change in C 2s population can give some effects on the X-ray absorption intensity excited by high-enegy X-ray photons.

\section{Concluding Remarks}

We have demonstrated that the X-ray absorption intensity is not simply the sum of free atomic X-ray absorption cross sections even in the high energy region. Two different effects are considered here. One is the many-body effects associated with the core-hole relaxation effects, and the other is the small tail from the valence photoabsorption which is in particular important for the systems with only light atoms. In the systems with heavy elements, next deepest edge tail is more influential than valence band tail. So that to avoid that complexity, $\mathrm{C}$ systems with only light elements are adequate to study the effects of the change in $2 \mathrm{~s}$ and $2 \mathrm{p}$ population. From the former effects we can estimate the morphology-dependent static dielectric function $\varepsilon(0)$. From the latter we can estimate the population on for example $\mathrm{C} 2 s$. From the experimental result, MWNT-7 has largest C $2 s$ population [4]. For the quantitative discussion we need very careful experimental setup.

In contrast to the high-energy region, different many-body effects play some important roles in addition to one-electron effects such as XANES in near 
edge region. Radiation field screening can contribute to the branching ratio of $L_{2} / L_{3}$ for transition metals which deviates from the simple statistical ratio [17], multi-atom resonant photoemission (MARPE) [18,19], and core-hole moving effects after the core excitation [20] .

\section{Acknowledgment}

The authors are grateful to useful comments on this manuscript by Dr. M. Shang and Dr. K. Niki. This work is supported by the Global COE Program (Advanced School for Organic Electronics) Chiba University. One of the author KK is supported by JST Exotic Nanocarbon Project, Japan Regional Innovation Strategy Program by the Excellence.

\section{References}

[1] H. Bethe and R. Jackiw, Intermediate Quantum Mechanics, 3rd ed. (Addison Wesley, 1986)

[2] J. Berkowitz, Atomic and Molecular Photoabsorption: Absolute Total Cross Sections, (Academic Press, 2002)

[3] B. S. Itchkawitz, J. P. Long, T. Schedel-Niedrig, M. N. Kabler, A. M. Bradshaw, R. Schlögl, W. R. Hunter, Chem. Phys. Lett. 243 (1995) 211.

[4] T. Fujimori, S. Tsuruoka, B. Fugetsu, S. Maruyama, A. Tanioka, M. Terrones, M. S. Dresselhause, M. Endo, K. Kaneko, Materials Express 1 (2011) 1.

[5] L. Hedin, Physica B 158 (1989) 344.

[6] L. Campbell, L. Hedin, J. J. Rehr, W. Bardyszewski, Phys. Rev. B 65 (2002) 064107.

[7] T. Fujikawa, J. Phys. Soc. Jpn. 68 (1999) 2444.

[8] T. Fujikawa, J. Synchrotron Rad. 8 (2001) 76.

[9] T. Fujikawa, K. Hatada, L. Hedin, Phys. Rev. B 62 (2000) 5387.

[10] R. Manne, T. Åberg, Chem. Phys. Lett. 7 (1970) 282.

[11] L. Hedin, J. Phys. Condens. Matter 11 (1999) R489.

[12] M. Kazama, H. Shinotsuka, M. Yamazaki, J. Adachi, A. Yagishita T. Fujikawa, J. Phys. Conference 190 (2009) 012048 1-4.

[13] M. Kazama, J. Adachi, H. Shinotsuka, M. Yamazaki, Y. Ohori, A. Yagishita, T. Fujikawa, Chem. Phys. 373 (2010) 261.

[14] T. Fujikawa, H. Arai, R. Suzuki, H. Shinotsuaka, L. Köver, N. Ueno, J. Elect. Spect. Relat. Phenom. 162 (2008) 146. 
[15] C-O. Almbladh, L. Hedin, E.E. Koch ( Eds.) Handbook on Synchrotron Radiation ( North-Holland), Vol.1b, p.607 .

[16] M. B. Trzhaskovskaya, V. I. Nefedov, V. G. Yarzhemsky, Atomic Data and Nuclear Data Table 77 (2001) 97.

[17] A. Ankudinov, A. I. Nesvizhskii, J. J. Rehr, Phys. Rev. B 67 (2003) 115120.

[18] M. Mannela, S. H. Yang, B. S. Mun, F. J. Garcia de Abajo, A. W. Kay, B. C. Sell, M. Watanabe, H. Ohldag, E. Ahlenholtz, A. T. Young, Z. Hussain, M. A. Van Hove, C. S. Fadley, Phys. Rev. B 74 (2006) 1651106.

[19] H. Arai T. Fujikawa, Phys. Rev. B 72 (2005) 075102.

[20] T. Fujikawa, J. Phys. Conf. 190 (2009) 012014 1-12. 